\title{
PICKUP WATER GROUP IONS AT COMET GRIGG-SKJELLERUP
}

\author{
A. J. Coates ${ }^{1}$, A. D. Johnstone ${ }^{1}$, D. E. Huddleston ${ }^{1, *}$, B. Wilken ${ }^{2}$, K. Jockers ${ }^{2}$, H. Borg ${ }^{3}$, E. Amata ${ }^{4}$, \\ V. Formisano $0^{4}$ M. B. Bavassano-Cattaneo ${ }^{4}$, J. D. Winningham ${ }^{5}$, C. Gurgiolo ${ }^{5}$ and F. M. Neubauer ${ }^{6}$
}

\begin{abstract}
The density and velocity distribution of cometary water group ions was measured by the Giotto spacecraft in the regions upstream and downstream of the "bow shock' at comet Grigg-Skjellerup. The results show that the distributions of ions are ring-like until quite close to the shock, the timescales for pitch angle and energy diffusion appear similar and the ion density follows a $r^{-2}$ dependence.
\end{abstract}

\section{Introduction}

The cometary encounters by spacecraft in the mid-1980s were with the relatively high activity comet Halley (neutral gas production rate $Q \sim 6.9-10 \times 10^{29} \mathrm{~s}^{-1}$ ) (Krankowsky et al, 1986; Huddleston et al, 1990) and the medium activity Giacobini-Zinner where $Q \sim 4 \times 10^{28} \mathrm{~s}^{-1}$ (Mendis et al, 1986). On 10th July 1992 the Giotto spacecraft encountered another comet, Grigg-Skjellerup (GS), which has a short period ( $\sim 5.1$ years) and is relatively inactive. For GS, $Q \sim 7.5 \pm 1.5 \times 10^{27} \mathrm{~s}^{-1}$ according to the first results from the Giotto plasma payload (Johnstone et al, 1993; Neubauer et al, 1993) and from ground-based observations (Jockers et al, 1993).

An overview of the JPA-IIS (Johnstone plasma analyser, implanted ion sensor) data including the identification of boundary crossings has been presented elsewhere (Johnstone et al, 1993). In this paper we study the distributions of cometary water group ions picked up by the solar wind using some of the techniques developed for comet Halley (Coates et al, 1989).

\section{Instrument and data analysis}

The IIS consists of a set of five spherical section electrostatic analysers followed by time of flight cells. Together these determine $E / q$ and $m / q$ for incoming ions. The five analysers sample different polar angles in the spacecraft frame. The overall energy range of the instrument is $86 \mathrm{eV}$ $86 \mathrm{keV}$ (Wilken et al, 1987) in 32 energy bins. The energy level is held for a spin (4s) and then is stepped to alternate levels for 16 spins up to the maximum energy; after this the intervening steps are sampled in the next 16 spins with energy decreasing. 32 spins or 128 seconds are therefore required for full coverage. One of the three data types produced by the instrument is used here, the '4D mode', where angular information is retained ( 16 azimuthal bins around $360^{\circ}$ in spin angle and 5 polar angle bins) and events are

\footnotetext{
${ }^{1}$ Mullard Space Science Laboratory, University College London, UK

${ }^{2}$ Max-Planck-Institut für Aeronomie, Germany

${ }^{3}$ IRF-Umea, Sweden

IFSI-CNR, Frascati, Italy

${ }^{5}$ Southwest Research Institute, San Antonio, Texas

${ }^{6}$ Institut für Geophysik und Meteorologie, Universität

Köln, Germany

"Present address Jet Propulsion Laboratory, Pasadena, California
}

Copyright 1993 by the American Geophysical Union.

Paper number 93GL00174

0094-8534/93/93GL-00174\$03.00 sorted into 5 mass groups. Here we use the first mass group (0-1.7 amu/q) to determine the solar wind density and velocity and we also use mass group 3 covering 6.5-20 $\mathrm{amu} / \mathrm{q}$ for the water group measurements.

Mass group 3 also includes the solar wind ions $\mathrm{Fe}^{9+}$ to $\mathrm{Fe}^{6+}$ which had unusually high density during the GS encounter (Johnstone et al, 1993). These ions were excluded from the present analysis by careful selection of the energy range to be included for each distribution. As the solar wind slows, the included energy range extends to lower energy to follow the water group ion population. An energy and angle dependent background matrix was subtracted from each measurement. In addition a threshold of 2 counts was used and the same factor 2.3 used at Halley was used to boost counts in polar bin 4 , on the basis of solar wind frame phase space densities at Halley (Coates et al, 1989). A higher microchannel plate voltage was used here than at the Halley encounter, and comparisons made on 9 July using both channel plate voltages show that the efficiency used in the Halley analysis should be multiplied by 2 for water group ions at Grigg-Skjellerup to compensate. As regards proton density, we pointed out (Johnstone et al, 1993) that the solar wind proton densities are preliminary estimates because of saturation effects in the IIS. Cross calibration with the Fast Ion Sensor at Halley and the $9 \mathrm{July}$ comparisons showed that multiplication by a factor 16.7 compared to the values obtained using the nominal IIS geometric factor gives a reasonable first estimate of density at this microchannel plate voltage and this correction is used both in the first results paper (Johnstone et al, 1993) and here.

The main analysis techniques used in this paper on the remaining counts are moment integrations and transformation of the measured phase space densities into a solar wind magnetic field aligned (SWB) frame from which $V_{\perp}-V_{\|}$distributions are obtained (for details of the analysis see Coates et al, 1989). The magnetic field values used here are 128 second averages of the magnetic field vector from the Giotto magnetometer sampled at the same time as the IIS measurements.

\section{Water group ion densities}

Figure 1 shows the radial profile of water group ion density for (a) the inbound pass and (b) the outbound pass. Examination of the energy distribution of the ions shows that the densities in the plot are underestimated for distances less than $\sim 5-6000 \mathrm{~km}$ from the nucleus since the energy of some of the water group ions goes below the energy range where the IIS calibration numbers are reliable. Beyond this distance the densities have been fitted to a straight line on the log-log plot. For the inbound pass values up to $300,000 \mathrm{~km}$ were used for the fit and for the outbound pass values up to $250,000 \mathrm{~km}$ were used. As can be seen from the fit parameters on the figures the basic radial dependence of the cometary ion density is close to $r^{-2}$ in each case, but with significant deviations.

The increases and decreases of the density with respect to $r^{-2}$ tend to be anticorrelated with changes in the angle $\alpha$ between the solar wind velocity and the magnetic field vectors. This can be seen in Figure 1 , where $\alpha$ is overplotted as a dashed line according to the right hand scale. One example is seen on the outbound pass at approximately $250,000 \mathrm{~km}$ where the density suddenly drops to effectively background levels, near to an increase in $\alpha$. 

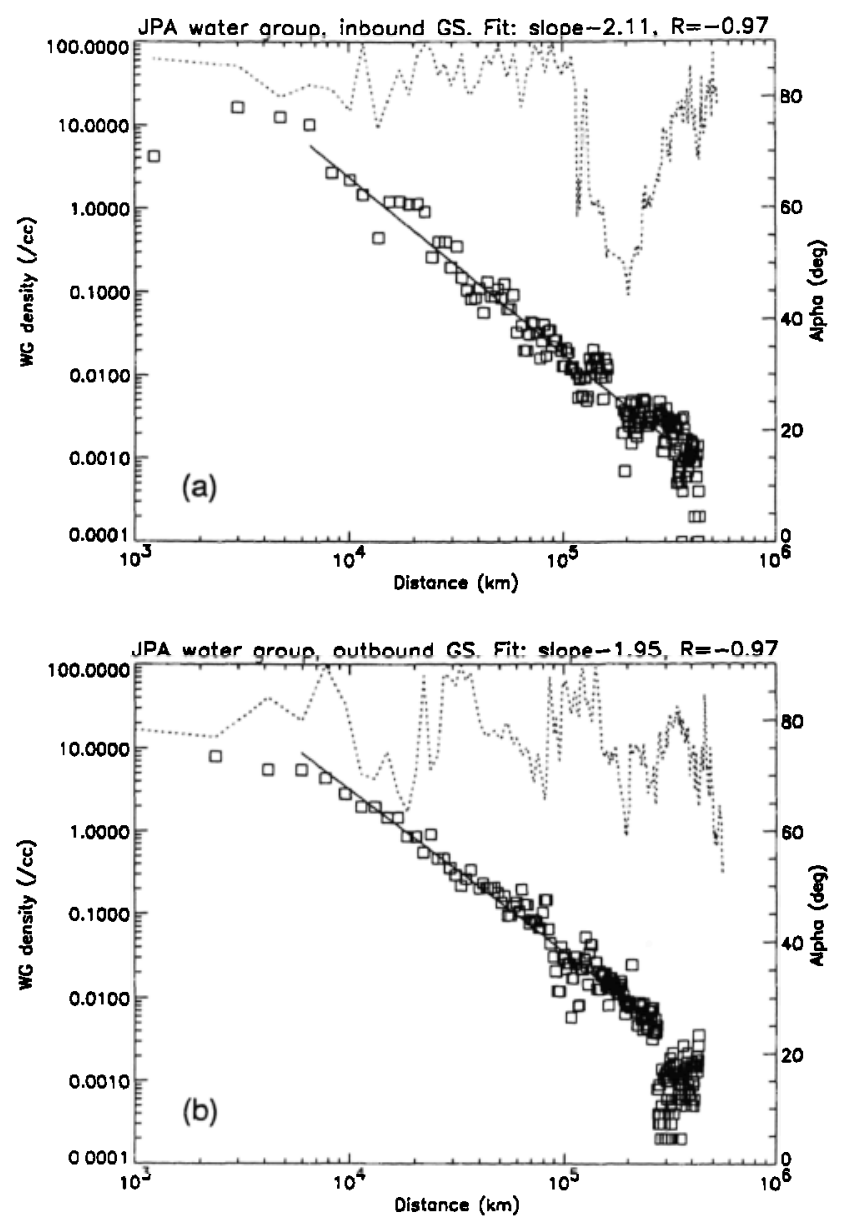

Fig. 1. Cometary ion density dependence on distance for (a) inbound pass and (b) outbound. The value of $\alpha$ is superimposed according to the right hand scale.

\section{Derived parameters}

Using the solar wind parameters and magnetic field data we have calculated some parameters relevant to the solar wind interaction in general and the ion pickup process in particular. These parameters are shown in Figure 2 which covers the distance range $350,000 \mathrm{~km}$ either side of closest approach (at 1518 SCET - spacecraft event time). Where necessary the measured density of solar wind protons was increased by $20 \%$ to allow for alpha particles and heavier ions (Johnstone et al, 1993). From the bottom the panels show the solar wind velocity $V$, Alfven speed $V_{A}$, Alfven Mach number $M_{A}, \alpha$ (calculated in the range $0-90^{\circ}$ ), and the maximum energies of a ring and shell distribution of water group ions in the spacecraft frame. Neglecting the neutral particle velocity with respect to the spacecraft these energies are $4 M E_{s w} \sin ^{2} \alpha$ and $4 M E_{s w}$, where $\mathrm{M}$ is the mass ratio between the pickup ions and protons (taken as 16 here) and $E_{s w}$ is the solar wind energy.

Figure 2 shows that $V_{A}$ is high throughout the encounter, consequently $M_{A}$ is unusually low at $\sim 3$ (but time dependent). The $\alpha$ angle is between $75^{\circ}$ and $90^{\circ}$ within $\pm 100,000$ $\mathrm{km}$ but is lower on average at larger distances. Consequently the maximum energy for the ring and shell are almost identical for more than 2 hours each side of closest approach $( \pm 100,000 \mathrm{~km})$ and the deviation elsewhere is larger, particularly between 1000-1230 SCET where $\alpha$ is lowest. Note that the central 2-3 points in these panels are overestimated because the solar wind peak moves lower than the lowest energy of the instrument, therefore the solar wind velocity should be lower for these few points.

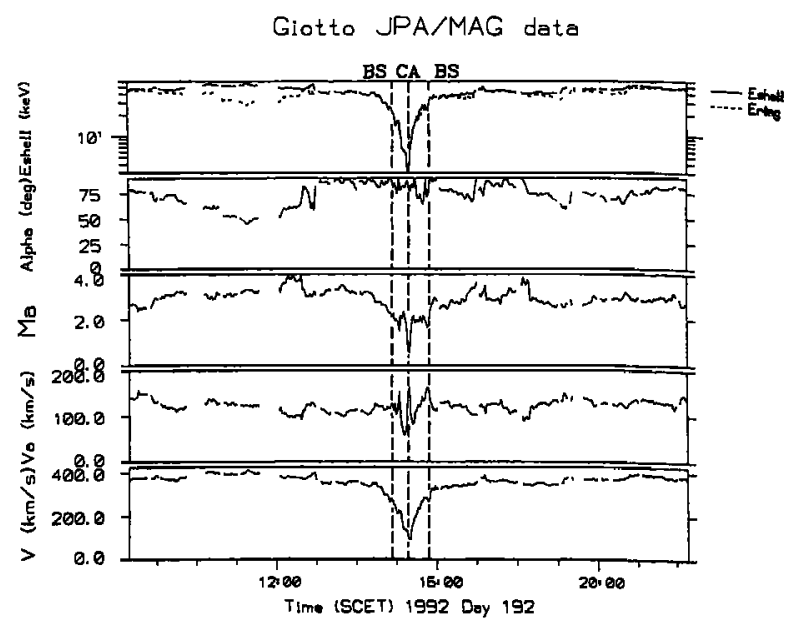

Fig. 2. Derived ion pickup parameters for $350,000 \mathrm{~km}$ each side of closest approach (CA).

\section{Ion distributions}

In Figure 3 we have overlaid the ring/shell predictions on an energy spectrogram of mass group 3 data which has been integrated over angle. The distance range of this plot is $\pm 350,000 \mathrm{~km}$. In this plot the lower two peaks $(0.8$ and $1.6 \mathrm{keV} / \mathrm{q}$ ) are due to saturation effects from the intense solar wind protons and alpha particles, the next peak ( $5 \mathrm{keV}$ ) is due to the solar wind iron ions mentioned be fore and the upper peak ( $\sim 40 \mathrm{keV}$ decreasing to $<1 \mathrm{keV}$ at closest approach) is due to water group ions. During the intervals when the ring and shell predictions deviate from each other (eg 1000-1230, 1800-2030) it can be seen that the maximum energy of the water group ions follows the ring prediction quite well. Closer to the comet it is diffcult to use this plot to diagnose the ring/shell nature of the distributions and angular information must be used. However it is clear from the plot that substantial enhancements of the fluxes above the ring/shell predictions are visible between approximately 1400 and 1615 SCET. Although some of the ions with energies up to $\sim 50 \mathrm{keV}$ may be due to pickup ions from upstream regions where the solar wind speed was higher (Thomsen et al, 1987) it is clear that further acceleration is necessary to explain the spectra we see.

Previously we have pointed out (Johnstone et al, 1993) that due to the geometry of the encounter and the masnetic field orientation, a ring distribution for $\alpha=90^{\circ}$ would appear in the central polar angle bin of the IIS. While this is observed in the $\alpha=90^{\circ}$ interval until quite close to the bow shock it is necessary to transform the measurements into a solar wind, magnetic field aligned frame to see this in detail and to characterise the distributions when $\alpha$ is not $90^{\circ}$. We present the results here as a sequence of $V_{\perp}-V_{1}$ plots where the solar wind velocity is the origin. In these plots the local (ring) pickup position is shown as a circled star and the simple shell, calculated using the local solar wind speed, is shown as a dashed semicircle in this plane (cf Coates et al, 1989). It should be remembered that each distribution is the sum of pickup ions produced locally with those from upstream. In this plane the moximum distance from the origin of all pickup ions is the upstream solar wind speed, ie $360 \pm 20 \mathrm{~km} / \mathrm{s}$ (Johnstone et al, 1993). Any ion seen further than this from the origin therefore has a higher energy than that available from the pickup process, probably due to energy scattering.

Figure 4 shows some $V_{\perp}-V_{\|}$distributions from the bound pass at Grigg-Skjellerup. In these plots phase spa density measurements are plotted at their transformed po sition in grey-scale; and measured zeroes are plotted as a 
GIOTTOH.JFA [14D LATA

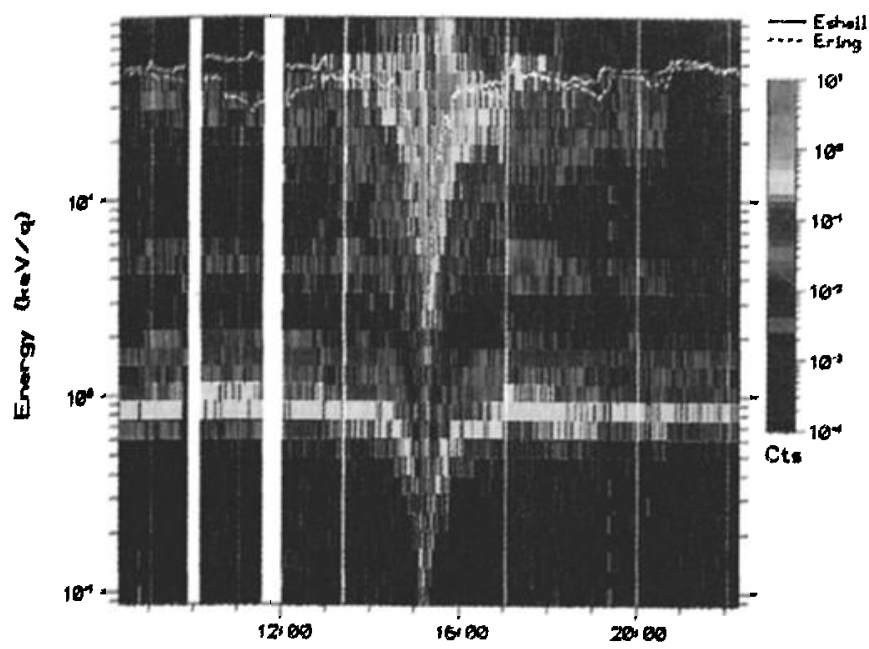

Fig. 3. Energy spectrogram of mass group 3 with ring (dashed line) and shell (solid line) predictions superimposed, for the same distance range as Figure 2.

light shade of grey. Regions with no measurement are left blank. Panel (a) shows a distribution which is quite ring-like and in (b) some limited pitch angle scattering has occured. Panel (c) shows evidence for some energy scattering while the pitch angle distribution is still quite narrow. Panel (d) shows the distribution at $42,000 \mathrm{~km}$ and here we see that significant pitch angle scattering has occurred and also some further acceleration of particles. Closer to the comet the distributions are broader still in pitch angle and energy. For comparison the 'bow shock' occurs at $\sim 1455$, in between panels (e) and (f).

Examples from the outbound pass are shown in Figure 5 , again close to the comet the distributions are broad in pitch angle and in energy. As with the inbound data a significant change is seen across the bow shock ( $\sim 1549$, in between panels (a) and (b)). Panel (c) is of interest because the distribution is 'one-sided' in pitch angle. Examination of spectrogram plots shows that this type of distribution persists from the outbound bow shock until approximately $1700 \operatorname{SCET}(85,000 \mathrm{~km})$; during this time
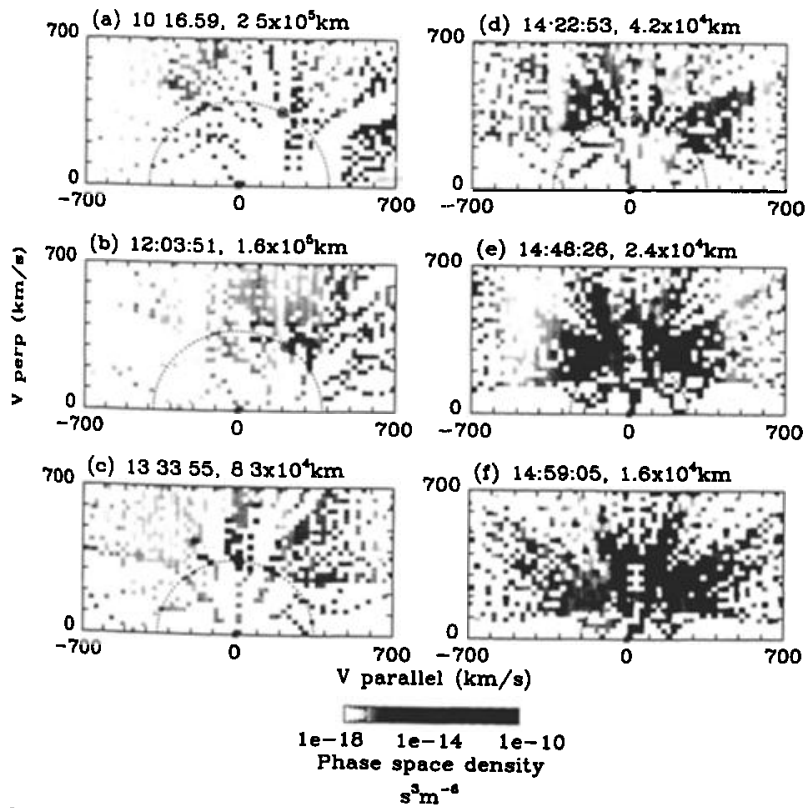

Fig. 4. $\quad V_{\perp}-V_{\| \mid}$distributions for some inbound examples.
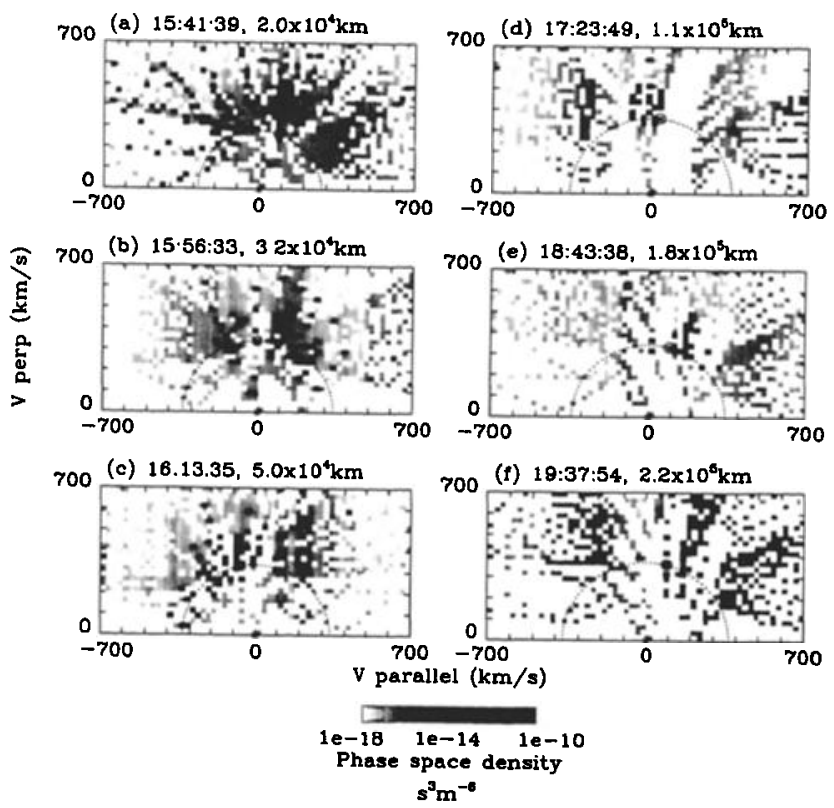

Fig. 5. $\quad V_{\perp}-V_{\|}$distributions for some outbound examples.

particles are seen in polar angles 3 and 4 of the IIS. Later, panels (d)-(f) once again show ring distributions.

Note that in the outer regions 5-distribution averages are used in Figures 4 and 5 because of the limited number of distributions in the encounter. For the two panels closest to the nucleus in each case individual distributions are shown because of the rapid velocity decrease of the solar wind there. These periods are both less than the 10distribution averages used at Halley (Coates et al, 1989) and consequently the noise levels are higher in the data shown here.

\section{Discussion}

The $r^{-2}$ dependence of cometary ion density is something of a surprise. The neutral density at a point in the coma is proportional to $r^{-2}$ multiplied by an exponential loss factor. At GS because the photoionization scale length is greater than the distance over which the ions are observed, the exponential factor is close to 1 throughout. Nevertheless one would expect the ions from upstream to add to the locally picked up ions and modify the ion density profile to a somewhat steeper form than $r^{-2}$ (Huddleston et al, 1990; Coates et al, 1990). For comparison the water group density at Halley varied as $r^{-3.7}$ outside the bow shock while the pickup proton density varied as $r^{-2}$ (Neugebauer et al, 1990). This difference was attributed to the different ionization scale lengths of the species.

Cometary ion density was affected by $\alpha$ at comet Halley for both protons and water group ions (see Neugebauer et al, 1990) due to the different directions of the guiding centres in the coma as discussed in Neugebauer et al, 1989a. At Giacobini-Zinner, the EPAS experiment found a positive correlation with $\alpha$ (Sanderson et al, 1986) although they could not cover the pickup ion peak.

The ion distributions show a ring like behaviour in the outer regions gradually broadening in pitch angle and energy. While there is not a precise 'boundary' where scattering occurs, some scattering is certainly evident by approximately $42,000 \mathrm{~km}$ inbound and $85,000 \mathrm{~km}$ outbound. We find that the average time spent by a pickup ion in the flow at these points (see Huddleston et al, 1992 for details) are 265 and 310 seconds respectively, much less than the same calculation yielded near to the bow shock at Halley where almost filled shells were seen (Coates et al, 1989). When the distributions do scatter it appears that 
pitch angle and energy diffusion occur at similar times. Indeed even when they are still basically ring-like, some of the distributions appear to be scattered to higher energy. This may be due to the high ratio of the Alfven speed to the solar wind speed (ie low $M_{A}$ ) here, since the square of this ratio is the ratio of energy to pitch angle diffusion in quasilinear theory as pointed out by Huddleston et al, 1993.

The one-sided distributions seen on the outbound leg between the bow shock $(\sim 20,000 \mathrm{~km})$ and $\sim 85,000 \mathrm{~km}$ are an interesting feature with no definite explanation at present. Some one-sided distributions of pickup protons were also seen at comet Halley during quasi-perpendicular periods (Neugebauer et al, 1989b). The aspects of the present encounter which are different to Halley which may contribute to these observations are:

1. the smaller size of features such as the bow shock which are the same order as the cometary ion gyroradius;

2. the high Alfven speed and lower solar wind Mach number at the encounter;

3. the $\alpha$ angle is near to $90^{\circ}$ for significant portions of this encounter.

Further analysis and modelling will need to take account of these facts and the relatively simple waves seen in the magnetic field throughout the encounter (Neubauer et al, 1993).

It is interesting to note that at the Earth's bow shock effects such as the energy scattered ring distributions and one-sided pitch angle distributions have been detected in cases where the Mach number was low (Sckopke et al, 1990).

Acknowledgments. We thank G.Paschmann for pointing out the possible similarity to distributions sometimes seen at the terrestial bow shock. The JPA experiment was supported by the UK Science and Engineering Research Council, the German Bundesministerium für Forschung und Technologie, NASA, the US Department of Energy, the Swedish Board for Space Activities and the Piano Spaziale Nazionale of the Consiglio Nazionale delle Ricerche, Italy. AJC acknowledges the support of the Royal Society, UK. FMN acknowledges support by the German Bundesministerium für Forschung und Technologie through DARA.

\section{References}

Coates, A.J., A.D. Johnstone, D.E. Huddleston, B. Wilken, $\mathrm{K}$. Jockers and K.-H. Glassmeier, Velocity space diffusion of pick-up ions from the water group at Comet Halley, J.Geophys.Res., 94, 9983-9993, 1989. (Correction 95, p4343, 1990).

Coates, A.J., B. Wilken, A.D. Johnstone, K. Jockers, K.-H. Glassmeier and D.E. Huddleston, Bulk properties and velocity distributions of water group ions at Comet Halley: Giotto measurements J.Geophys.Res., 95, 10249-10260, 1990.

Huddleston, D.E., A.D. Johnstone and A.J. Coates, Determination of comet Halley gas emission characteristics from mass loading of the solar wind, J.Geophys.Res., 95, 21-30,
1990 .

Huddleston D.E., A.D. Johnstone and A.J. Coates, Massloading and velocity diffusion models for heavy pickup ions at comet Grigg-Skjellerup (abstract), EOS Trans. AGU, 73(43), Fall Meeting Suppl., p438, 1992.

Huddleston, D.E., A.D. Johnstone and A.J. Coates, Diffusion of cometary pickup ions on bispherical characteristics, in Proc of 4 th COSPAR colloquium on 'Critical problems in the plasma environment of comets and other non-magnetized and weakly magnetized bodies', Ann Arbor, Michigan, in press, 1993.

Jockers, K., N.N. Kiselev, H. Boehnhardt and N. Thomas, $\mathrm{CN}, \mathrm{C}_{2}$ and dust observed in comet $\mathrm{P} / \mathrm{Grigg}$-Skjellerup from the ground eight hours after the Giotto encounter, Astron. Astrophys., in press, 1993.

Johnstone, A.D., A.J. Coates, D.E. Huddleston, K. Jockers, B. Wilken, H. Borg, C. Gurgiolo, J.D. Winningham and E. Amata, Observations of the solar wind and cometary ions during the encounter between Giotto and comet Grigg-Skjellerup, Astronomy and Astrophysics, in press, 1993.

Krankowsky, D., P. Lämmerzahl, I. Herrwerth, J. Woweries, P. Eberhardt, U. Dolder, U. Herrmann, W. Schulte, J.J. Berthelier, J.M. Iliano, R.R. Hodges and J.H. Hoffman, In situ gas and ion measurements at comet Halley, Nature, 321,
$326-329,1986$.

Mendis, D.A., E.J. Smith, B.T. Tsurutani, J.A. Slavin, D.E. Jones and G.L. Siscoe, Comet-solar wind interaction: dynamical length scales and models, Geophys. Res. Letters, $13,239-242,1986$.

Neubauer, F.M., H. Marschall, M. Pohl, K.-H. Glassmeier, G. Musmann, F. Mariani, M.H. Acuña, L.F. Burlaga, N.F. Ness, M.K. Wallis, H.U. Schmidt and E. Ungstrup, Preliminary results from the Giotto Magnetometer Experiment during the $P$ / Grigg-Skjellerup encounter, Astronomy and Astrophysics, in press, 1993.

Neugebauer, M., B.E. Goldstein, H. Balsiger, F.M. Neubauer, R. Schwenn and E.G. Shelley, The density of cometary protons upstream of comet Halley's bow shock, J.Geophys.Res., 94, 1261-1269, 1989a.

Neugebauer, M., A.J. Lazarus, H. Balsiger, S.A. Fuselier, F.M. Neubauer and H. Rosenbauer, The velocity distributions of cometary protons picked up by the solar wind, J.Geophys.Res., 94, 5227-5239, 1989b.

Neugebauer, M., A.J. Coates and F.M. Neubauer, Comparison of picked up protons and water group ions upstream of comet Halley's bow shock, J. Geophys. Res., 95, 18745-18753, 1990.

Sanderson, T.R., K-P. Wenzel, P. Daly, S.W.H. Cowley, R.J. Hynds, E.J. Smith, S.J. Bame and R.D. Zwickl, The interaction of heavy ions from comet P/Giacobini-Zinner with the solar wind, Geophys.Res.Letters, 13, 411-414, 1986.

Sckopke, N., G. Paschmann, A.L. Brinca, C.W. Carlson and H. Iüh, Ion thermalization in quasi-perpendicular shocks involving reflected ions, J.Geophys. Res., 95, 6337-6352, 1990.

Thomsen, M.F., W.C. Feldman, B. Wilken, K. Jockers, W. Stüdemann, A.D. Johnstone, A.J. Coates, V. Formisano, E. Amata, J.D. Winningham, H. Borg, D. Bryant and M.K. Wallis, In-situ observations of a bi-modal ion distribution in the outer coma of comet Halley, Astron. Astrophys., 187, $141-148,1987$.

Wilken, B., W. Weiss, W. Stüdemann and N. Hasebe, The Giotto implanted ion spectrometer(IIS): Physics and technique of detection, J. Phys. E Sci. Instrum., 20, 778-785, 1987.

E. Amata, M. B. Bavassano-Cattaneo, V. Formisano, IFSICNR, Via G.Galilei, C.P.27, 00044 Frascati, Italy

H. Borg, Swedish Institute of Space Physics (IRF), University of Umea, S-901 87 Umea, Swreden

A.J.Coates, A.D.Johnstone, D.E.Huddleston,Mullard Space Science Laboratory, University College London, Holmbury St Mary, Dorking RH5 $6 \mathrm{NT}$, UK

F. M. Neubauer, Institut für Geophysik und Meteorologie, Universität Köln, W-5000 Köln 41, Germany

B. Wilken, K.Jockers, Max Planck Institut für Aeronomie, W-3411 Katlenburg-Lindau, Germany

J. D. Winningham, C. Gurgiolo, Southwest Research Institute, PO Drawer 28510, San Antonio, Texas 78228-0510, USA

(Received December 2, 1992; revised January 11, 1993; accepted January 11, 1993.) 